\title{
Time Constants for Nonstationary Arcs
}

\author{
RICHARD L. PhILliPS * \\ The University of Michigan, Ann Arbor, Michigan
}

Received August 17, 1967

\begin{abstract}
The time constants associated with the initial deviations of an arc column from a static siate have been studied analytically. The basic approach is to use a Taylor series expansion in powers of time of the energy balance equation. It is assumed that the arc gas is optically thin and is in local thermal equilibrium. Initially the effects of radial gas flow (which nust exist for dynamic arcs) are neglected, but this simplification is later relaxed. The initial conditions are given by the properties of a cylindrically symmeiric, wall-stabilized DC positive column. The interrupted (freely decaving) and the step-modulated arcs are considered, and initial time constants for conductance, electric field, and heat flux potential are computed. For numerical results the best available values of thermodynamic and transport properties have been used.
\end{abstract}

\section{Introduction}

In those fields where the behavior of a non-stationary arc plays an important role, the concept of a time constant is useful for characterizing the rate of change of certain properties. Depending upon which variable one is interested in, it is possible to define time constants for temperature, electric field strength, radiative emission, and electrical conductance. For example, in $\mathrm{AC}$ arc heater technology ${ }^{1,2}$ one wishes to know the characteristic frequency (inverse time constant) for conductance variations of the arc in order to evaluate its role as a circuit element. Also, since the early 1930 's, the designers of switchgear have found temperature and conductance time constants to be useful in describing the effectiveness of a circuit breaker ${ }^{3,4}$. Finally, recent interest in the determina-

* Department of Aerospace Engineering, University of Michigan, Ann Arbor, Michigan (USA). The research reported here was supported in part by the Institute of Science and Technology, The University of Michigan and the National Science Foundation under Grant No. GK 1451. The support of both agencies is gratefully acknowledged.

${ }^{1}$ Phillits, R.L.: Aerospace Research Lab Report 64-9, 1964.

2 PenLliss, R.L.: The behavior of dynamic electric arcs. Thesis, University of Michigan (1964).

3 MAYR, O.: Arch. Elektrotechnik 37, 588 (1943).

${ }^{4}$ CASSn:, A. M.: CIGRE (Paris), 1 (1939). 
tion of high temperature transport properties from pulsed arcs ${ }^{5}$, gives rise to a need for the time constant of electric field variation.

The arc geometries and boundary conditions that occur in the above named areas are many and varied. The main purpose here, however, is to examine the relationship between time constants and plasma material properties, so we study the well defined problem of a cylindrical positive column produced by a wall stabilized electric arc. Furthermore, with the aim of obtaining analytical results, the present work is concerned with the variation of plasma properties for only the first moments after interruption or step current modulation of a DC arc.

The problem posed above has been studied before, most notably by FRIND $^{6}$. In all previous work ${ }^{3,4,6}$, however, including the 1965 papers of EDELS ${ }^{7}$ and KRIZHANSKII ${ }^{8}$, either a structureless arc was considered or simplified material properties were used. As a result it has not been possible to predict, for example, the time constant behavior that was observed experimentally by CHRISTMANN, FRIE, and HERTz ${ }^{9}$ or KIMBLIN and EDELS ${ }^{10}$, showing the occurrence of two different rates of conductance decay. In the present work analytical expressions for various time constants (heat flux potential, conductance, and electric field strength) are obtained that can be evaluated using the best available, actual material properties.

A minimum number of simplifying assumptions are made. The energy balance used herein assumes the existence of local thermal equilibrium and an optically thin arc gas. The effects of radial gas flow are included in the present study. Conservation of mass requires the existence of such a flow to compensate for temporal density variations, and it is clear that the ensuing radial convection of heat will influence the transient temperature distribution. The present analysis is concerned with an infinitely long cylindrical arc, where there are no axial gradients. If one considers, however, a model where end effects are permitted it is necessary to allow for the existence of an induced axial flow. This point is discussed more fully in Sec. 6 where it is shown that in certain circumstances axial as well as radial convection can be ignored.

In order to show the relation of the present results to previous work, the governing equations are formulated in terms of the heat flux potential. Later it is shown that a more suitable variable exists, one that permits ready comparison of the present theory with experimental results.

\footnotetext{
${ }^{5}$ WATSON, V.R.: Private communication (1967).

${ }^{6}$ FrIND, G.: Z. angew. Phys. 12, 231 (1960).

${ }^{7}$ Edels, H., and F.H. FenLon: Brit. J. Appl. Phys. 16, 219 (1965).

${ }^{8}$ KrIZHANSKII, S. M.: Sov. Phys.-Tech. Phys. 10, 1450 (1966).

9 Chrustmann, H., W. Frie u. W. Hertz: Z. Physik 203, 372 (1967).

${ }^{10}$ Kimblin, C., and H. Edels: Brit. J. Appl. Phys. 17, 1607 (1966).
} 


\section{The Fundamental Equations}

If one assumes the existence of local thermodynamic equilibrium (LTE), an optically thin arc gas, that baro- and thermal diffusion are unimportant, and at most a velocity in the radial direction, it is possible to write the cylindrically symmetric conservation equations in the form. ${ }^{2}$

$$
\begin{gathered}
R \frac{\partial \rho}{\partial t}+\frac{1}{r} \frac{\partial}{\partial r}(\rho r v)=0, \\
R^{2} \rho \frac{D v}{D t}+R \frac{\partial p}{\partial r}-\frac{\partial}{\partial r}\left\{\mu\left[2 \frac{\partial v}{\partial r}-\frac{2}{3} \frac{1}{r} \frac{\partial}{\partial r}(r v)\right]\right\} \\
-\frac{2}{r} \mu\left(\frac{\partial v}{\partial r}-\frac{v}{r}\right)=0, \\
R^{2} \rho \frac{\partial h}{\partial t}+R v \rho \frac{\partial h}{\partial r}-R^{2} \frac{D p}{D t}-\frac{1}{r} \frac{\partial}{\partial r}\left(r \vec{\kappa} \frac{\partial T}{\partial r}\right) \\
-R^{2}\left(\sigma E^{2}-u\right)-R^{2} \Phi=0, \\
2 \pi R^{2} E \int_{0}^{1} r \sigma(T, p) d r=I, \\
0 \leqq r \leqq 1, \quad T(1, t)=T_{w} .
\end{gathered}
$$

Eqs. (1), (2), and (3) express conservation of mass, momentum, and energy, while Eq. (4) is the appropriate form of OHm's law. In the above $\sigma, T, \rho, h$, and $u$ are the electrical conductivity, temperature, mass density, enthalpy, and radiation density, respectively. The diffusive contribution to energy transfer is combined with the ordinary thermal conductivity to give a total thermal conductivity, $\bar{\kappa}$. Other symbols that appear are $\Phi$, the viscous dissipation function, $v$, the radial velocity, $\mu$, the viscosity, and $p$ the pressure. The plasma is confined in a tube of radius $R$ with specified wall temperature $T_{w}$. The radial variable is normalized with respect to $R$ so that $0 \leqq r \leqq 1$ is the spatial domain of interest. In Eq. (4) $E$ and $I$ represent the electric field and current, both time dependent but not functions of $r$.

In what follows all transport properties are allowed to vary arbitrarily with temperature but because only small pressure variations will occur the pressure dependence of these coefficients is neglected. Then the heat flux potential $S$ can be introduced by

$$
S(T)=\int_{T_{\mathrm{ref}}}^{T} \bar{\kappa}(x) d x,
$$


allowing easy comparison of subsequent solutions with those of previous authors. When $S$ rather than $T$ and $h$ is used as the dependent variable in the energy equation, two additional symbols appear. These are $C_{p}$, the specific heat at constant pressure and the thermal diffusivity, defined by

$$
\lambda=\bar{\kappa} j \rho C_{p} .
$$

To complete the set of conservation equations one requires an equation of state, given here in the functional form

$$
p=p(S, \rho) .
$$

In later sections it will be necessary to use Eq. (5) to replace pressure derivatives in the conservation equations. In this regard the following relation will be useful

$$
d p=\left(\frac{\partial p}{\partial S}\right)_{\rho} d S+\left(\frac{\partial p}{\partial \rho}\right)_{S} d \rho
$$

In the next section the conservation equations are greatly simplified by assuming a constant pressure column, in line with previous investigators. Later, when the effects of radial convection are introduced, the constant pressure assumption is relaxed.

\section{The Step-Modulated Arc without Convection (Including Interruption)}

The wall stabilized pulsed arc has been studied by a number of investigators as a means of obtaining time constants for various gases (YoON and SPINDLE ${ }^{11}$, FRIND $^{6}$ ). This method has the advantage over the free decay procedure (which is a special case of step-modulation) that the electric field can be easily measured, rather than using the impulse probe method ${ }^{9}$ to measure conductance. It is shown, however, that for given initial conditions the time constants obtained by these two methods are directly related.

Previous analyses of this problem have proceeded from the assumption of constant pressure. Strictly speaking, this implies no radial convection, which in turn obviates any further need for either the momentum or continuity equation. In the step-modulated arc one has a situation where a discharge that is being sustained by a steady current $I_{0}$ suddenly has imposed upon it a new, steady current $I_{n}$. At first the electric field is determined by the new current and the original conductance, but as the heat flux potential changes, $E$ approaches its new DC level. The constant pressure versions of Eq. (3) and (4) that govern this transient

\footnotetext{
11 Yoon, K.H., and H. E. SPINDLE: Trans. Am. Inst. Elect. Engrs. 77, 1634 (1959).
} 
process are

$$
\begin{gathered}
\frac{1}{r} \frac{\partial}{\partial r}\left(r \frac{\partial S}{\partial r}\right)+R^{2}\left(\sigma E^{2}-u\right)=\frac{R^{2}}{\lambda(S)} \frac{\partial S}{\partial t}, \quad 0 \leqq r \leqq 1, \quad t>0, \\
2 \pi R^{2} E \int_{0}^{1} r \sigma(S) d r=I_{n}, \quad S(1, t)=0 .
\end{gathered}
$$

The initial conditions, corresponding to $I=I_{0}$, are obtained from a solution of Eq. (7) with the transient term set to zero and with $I_{n}$ replaced by $I_{0}$ in Eq. (8). Properties pertaining to this initial state will be denoted by the subscript ()$_{0}$.

It is not possible to obtain a general solution to $\mathrm{Eq}$. (7) because $\lambda, \sigma$, and $u$ are all non-linear functions of $S$. It is of interest, however, to examine the behavior of the thermal and electrical variables shortly after the current has been modulated, i.e. for small time. We assume, therefore, that

$$
S(r, t) \cong S_{0}(r)+S^{\prime}(r, t)
$$

where $\left|S^{\prime} / S_{0}\right| \ll 1$. For the material properties one writes, for example,

$$
\sigma(S) \cong \sigma\left(S_{0}\right)+\left(\frac{d \sigma}{d S}\right)_{S_{0}}\left(S-S_{0}\right)
$$

with similar relations for $\lambda$ and $u$. For the electric field, since one is interested in times $t \geqq 0+$, we have

$$
E(t)=\frac{I_{n}}{I_{0}} E_{0}+E^{\prime}(t)
$$

By using the above expansions about the initial state, the governing equations can be linearized, leaving one with a linear partial differential equation for $S^{\prime}$. Even this equation cannot be solved analytically, however, because it will contain complicated variable coefficients. A method by which one can avoid solving this equation numerically, and yet learn much about arc behavior, involves representing $S^{\prime}(i, t)$ as a power series in $t$ with coefficients that are functions of $r$. One writes, then

$$
S^{\prime}(r, t) \cong S_{1}(r) t+S_{2}(r) t^{2}+O\left(t^{3}\right)
$$

By inserting this assumed form into Eq. (7), and equating the coefficients of like powers of $t$, one obtains for the first coefficient the equation

$$
\frac{1}{\ddot{r}} \frac{d}{d r}\left(r \frac{d S_{0}}{d r}\right)+R^{2}\left[\sigma\left(S_{0}\right)\left(\frac{I_{n}}{I_{0}}\right)^{2} E_{0}^{2}-u\left(S_{0}\right)\right]=\frac{R^{2}}{\lambda\left(S_{0}\right)} S_{1}(r) .
$$


Similar equations can be written down immediately for $S_{2}, S_{3}$, etc. From the stationary form of Eq. (7) it is seen that

$$
\frac{1}{r} \frac{d}{d r}\left(r \frac{d S_{0}}{d r}\right)+R^{2}\left[\sigma\left(S_{0}\right) E_{0}^{2}-u\left(S_{0}\right)\right]=0,
$$

leading to the result

$$
S_{1}(r)=\lambda\left(S_{0}\right) \sigma\left(S_{0}\right) E_{0}^{2}\left[\left(\frac{I_{n}}{I_{0}}\right)^{2}-1\right]
$$

We are now in a position to evaluate several types of time constants for the modulated arc. From an electrical standpoint the variation of column conductance and electric field strength is of interest. The former is defined by

$$
G(t)=2 \pi R^{2} \int_{0}^{1} r \sigma(S) d r .
$$

Using the expansion procedure outlined above, one can write

$$
G(t) \cong 2 \pi R^{2}\left[\int_{0}^{1} r \sigma\left(S_{0}\right) d r+t \int_{0}^{1} r\left(\frac{d \sigma}{d S}\right)_{S_{0}} S_{1}(r) d r+O\left(t^{2}\right)\right] .
$$

The coefficient $S_{1}(r)$ is obtained from Eq. (9) so that we may write

$$
\begin{aligned}
G(t)= & G_{0}\left\{1+\frac{2 \pi R^{2} E_{0}^{2}}{G_{0}}\left[\left(\frac{I_{n}}{I_{0}}\right)^{2}-1\right] t\right. \\
& \left.\cdot \int_{0}^{1} r\left(\frac{d \sigma}{d S}\right)_{S_{0}} \sigma\left(S_{0}\right) \lambda\left(S_{0}\right) d r+O\left(t^{2}\right)\right\},
\end{aligned}
$$

where $G_{0}$ is the conductance in the initial state. Up to this point no specific model for $\lambda, \sigma$, or $u$ has been assumed, so the analysis is quite general.

By taking the derivative of $\ln G$ (from Eq. (10)) and letting $t \rightarrow 0$, one obtains the inverse of the initial time constant for conductance variation, i.e. the time constant that applies at the moment of modulation and shortly thereafter. The resulting expression is

$$
\begin{aligned}
& \left(\frac{d \ln G}{d t}\right)_{t=0}=\theta_{g}^{-1} \\
& \quad=\frac{2 \pi R^{2} E_{0}^{2}}{G_{0}}\left[\left(\frac{I_{n}}{I_{0}}\right)^{2}-1\right] \int_{0}^{1} r\left(\frac{d \sigma}{d S}\right)_{S_{0}} \sigma\left(S_{0}\right) \lambda\left(S_{0}\right) d r .
\end{aligned}
$$

Since we have specialized to $t=0$, Eq. (11) is exact. Clearly, it is possible to obtain values for the time constant for subsequent values of time by using the recursion relations for $S_{2}, S_{3}$, etc. According to Eq. (11) the 
initial rate of variation of conductance is determined by a weighted integral over the arc cross-section of $\lambda, \sigma$, and the slope of the $\sigma(S)$ curve. The initial conditions are felt through $S_{0}(r)$, as well as $E_{0}$ and $G_{0}$.

For the variation of heat flux potential after modulation a procedure similar to that given above yields the result

$$
\left(\frac{\partial \ln S}{\partial t}\right)_{t=0}=\theta_{s}^{-1}=\left[\left(\frac{I_{n}}{I_{0}}\right)^{2}-1\right] E_{0}^{2} \frac{\lambda\left(S_{0}\right) \sigma\left(S_{0}\right)}{S_{0}(r)} .
$$

Unlike $\theta_{g}$, this time constant is a function of $r$.

Since the electric field is readily measured in a step-modulation experiment, one should relate the variation of $E$ to the conductance time constant. It is convenient to obtain an expression for the difference between $E(t)$ and the final electric field $E_{n}$. This is done to facilitate comparison with the results of a study still in progress where the time constant for approach to the final state is being examined. If one writes the expansion for $E(t)$ in the form

$$
E(t)=\frac{I_{n}}{I_{0}} E_{0}+E_{1} t+O\left(t^{2}\right),
$$

then immediately after modulation we find

$$
\left[\frac{d \ln \left(E-E_{n}\right)}{d t}\right]_{t=0}=\theta_{e}^{-1}=\frac{E_{1}}{\left(\frac{I_{n}}{I_{0}}\right) E_{0}-E_{n}} .
$$

From OHм's law in Eq. (8) we find

$$
E_{1} G_{0}+2 \pi R^{2}\left(\frac{I_{n}}{I_{0}}\right) E_{0} \int_{0}^{1} r\left(\frac{d \sigma}{d S}\right)_{S_{0}} S_{1}(r) d r=0,
$$

leading to the result

$$
\theta_{e}^{-1}=-\theta_{g}^{-1}\left(\frac{I_{n} / I_{0}}{\frac{I_{n}}{I_{0}}-\frac{E_{n}}{E_{0}}}\right) .
$$

It is evident from Eq. (13) that the time constant for electric field variations is related to the conductance time constant by a function of DC arc parameters. If the electric field were not referred to its terminal value, Eq. (13) would simply be $\theta_{e}=\theta_{g}$. Here it should be emphasized that all of these time constants apply only to the first stages of current modulation. The determination of terminal time constants is considerably more difficult.

Within the framework of the above theory, arc interruption becomes simply a special case of current modulation, namely $I_{n}=0$. There is no 
electric field to vary in that case, but $\theta_{g}$ and $\theta_{s}$ in Eq. (11) and (12) reduce to their appropriate free decay forms. It is easily seen, in fact, that the time constants for step modulation and free decay differ only by the factor $\left(I_{n}^{2} / I_{0}^{2}-1\right)^{\star}$. In the next section the interruption theory will be applied to a particular analytical arc model and the results compared with a numerical computation in Section 6.

\section{Application to Analytical Models}

It is interesting to apply the results of the interruption theory to some analytically tractable arc models which have been presented in the literature $^{12,13}$. For many gases the electrical conductivity has the dependence upon heat flux potential that is shown in Fig. 1. The curves $(a),(b)$, and $(c)$

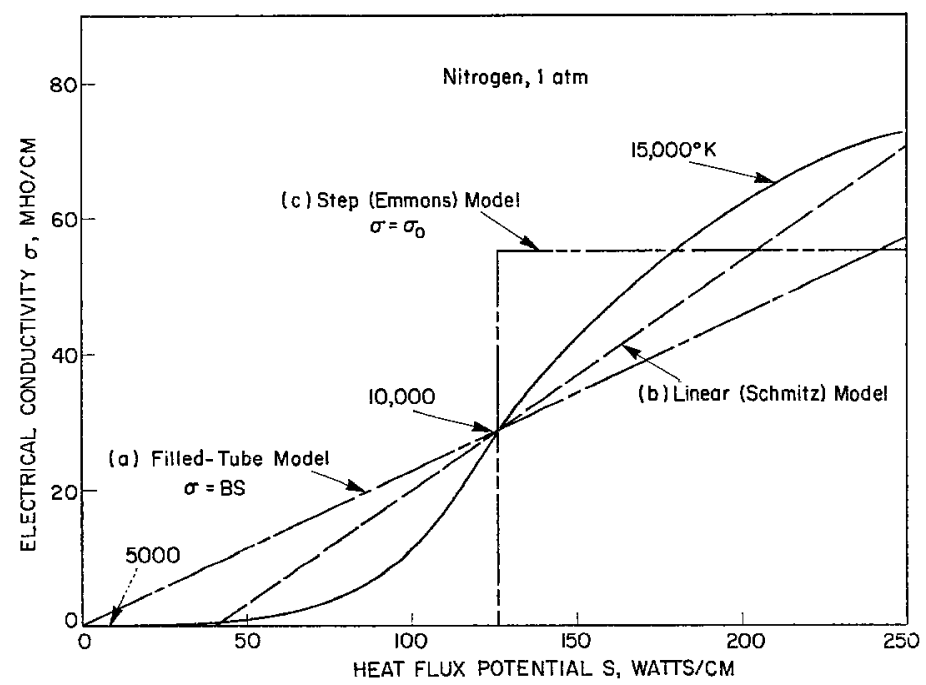

Fig. 1. Nitrogen electrical conductivity and approximate models

are the various approximations to the real behavior that have been used in obtaining analytical solutions to the Elenbaas-Heller equation, the stationary form of Eq. (7). Radiation is neglected in all cases. Curve (a) leads to the filled-tube model discussed by EDELS and FENLON ${ }^{7},(b)$ to the SCHMitz ${ }^{14}$-MAECKeR ${ }^{15}$ Bessel model, and $(c)$ to the EMmons ${ }^{13}$

* This result has been independently derived by H. Edels and E. GRAFFMANN, University of Liverpool for an arc with a linear $\sigma(S)$ behavior.

12 UhLENBUSCH, J.: Zur Theorie und Berechnung stationärer und quasistationärer zylindrischer Lichtbogen. Thesis, Aachen (1962).

${ }^{13}$ Emmons, H., and R. Land: Phys. Fluids 5, 1489 (1962).

${ }^{14}$ SCHMTTZ, G.: Z. Naturforsch. 5a, 571 (1950).

15 MAECKER, H.: Z. Physik 157, 1 (1959). 
discontinuity model. Only the Emmons model will be considered in detail here since the complete solutions for cases $(a)$ and $(b)$ can be found in the literature ${ }^{7,15}$. For instance, by using the solution of EDELS and FENLON ${ }^{7}$ for $S_{0}(r)$ and assuming that $\lambda\left(S_{0}\right)=\lambda_{0}$, a constant (the usual simplification in previous studies), one obtains from Eq. (11) for $I_{n}=0$ the relation

$$
\theta_{g}=\frac{R^{2}}{\beta_{1}^{2} \lambda_{0}} .
$$

Here $\beta_{1}=2.405$, the first zero of the Bessel function. For this "constant radius" arc it turns out that the time constant for heat flux potential decay also has the above value, independent of radius. This is the time constant for classical heat conduction, i.e. a solid cylinder whose temperature is decaying from some initial value. More sophisticated arc models, for example, where the radius of the conducting region is allowed to vary, have a heat flux potential time constant that differs from that for conductance.

In order to amplify the latter statement consider the second approximation to $\sigma(S)$, curve $(b)$. Here it is assumed

$$
\begin{array}{ll}
\sigma=0, & S \leqq S_{c}, \\
\sigma=B\left(S-S_{c}\right), & S \geqq S_{c} .
\end{array}
$$

In this case the solution to the stationary state equation has two parts, one in the conducting region $0 \leqq r \leqq r_{c}$ and another in the annular nonelectrical region, $r_{c} \leqq r \leqq 1$. Again, assuming constant thermal diffusivity, one obtains with the aid of MAECKER's ${ }^{15} S_{0}(r)$ the expression

$$
\theta_{\mathrm{g}}=R^{2} r_{c}^{2} / \beta_{1}^{2} \lambda_{0} .
$$

This model for the electrical conductivity endows $\theta_{g}$ with a current dependence, felt through the initial radius of the conducting zone. The initial time constant for $S$ decay at the center of the arc, given by

$$
\left.\theta_{s}\right|_{r=0}=\theta_{g}\left(1+\frac{2 \beta_{1} J_{1}\left(\beta_{1}\right) S_{c}}{E_{0} I_{0}}\right)
$$

can differ significantly from $\theta_{g}$. For a low power arc the difference can be nearly a factor of two.

The time constant for conductance decay given in Eq. (15) is identical to the value found by FRIND ${ }^{6}$, who indicated that it applied during the entire decay process. Actually, it is the proper value only immediately after interruption; for later times the conductance decays at a different rate. This can be seen from Fig. 2, where $G(t)$ is plotted for the entire 
free recovery period of an interrupted arc. The results were obtained by EDELS and FenLON ${ }^{16}$ by numerically integrating Eq. (7), using the present $\sigma(S)$ model. The curve indicates that $\theta_{g}$ decreases (the arc decays more rapidly) as time progresses. This is contrary to experimental evidence ${ }^{9}$.

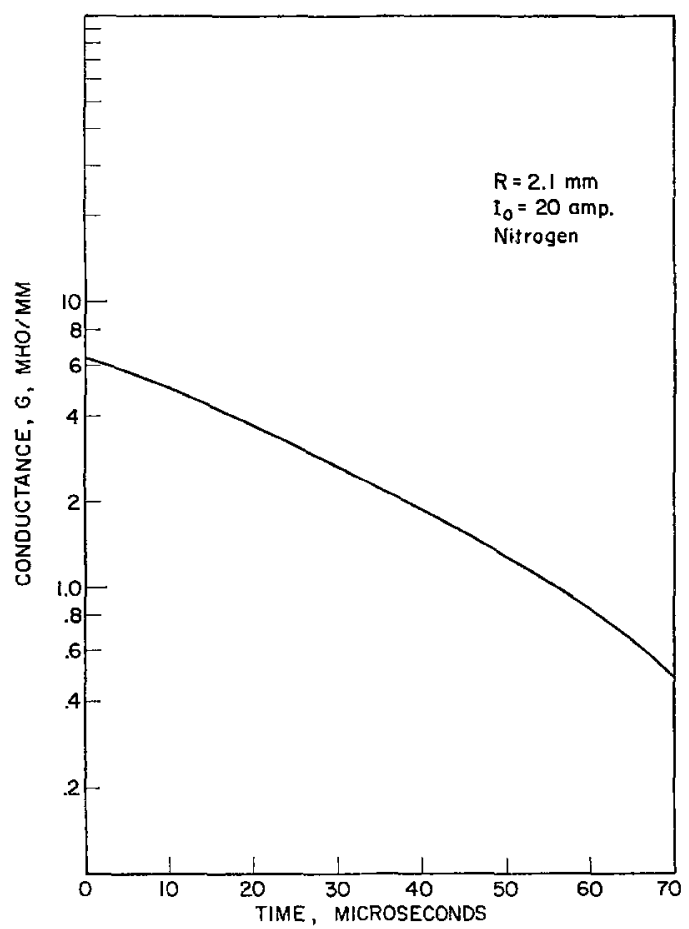

Fig. 2. Conductance variation for an interrupted arc (model b)

FRIND ${ }^{6}$ first showed that the temperature decay of an interrupted arc is represented by a whole spectrum of time constants. The value of $\theta_{s}$ given in Eq. (16) is in agreement with those findings, although not explicitly. It can be shown that Eq. (16) actually represents the summation of the infinite series solution found by FRIND ${ }^{6}$ and extended by PHILLIPS ${ }^{17}$.

EMMONs ${ }^{13}$ was the first to use a $\sigma(S)$ model that is capable of predicting both the rising and falling parts of the voltage-current characteristic for a wall-stabilized arc. With models $(a)$ and $(b)$ the arc characteristic can never exhibit a rising, ohmic behavior. For this reason it is interesting

${ }^{16}$ Edfis, H., and F.H. Fenlon: Proc. I.E.E. 110, 2082 (1963).

17 PHILliPs, R. L.: Proc. I.E.E. 113, 717 (1966). 
to study model (c), which can be represented by

$$
\sigma=B S_{c} U\left(S-S_{c}\right),
$$

where $U$ is the unit step function and $B$ and $S_{c}$ are appropriate constants, generally different from those used in the other models. The differential equation that determines the initial conditions is obtained from Eq. (7). With no radiation and with the transient term set to zero this equation becomes

$$
\frac{1}{r} \frac{d}{d r}\left(r \frac{d S_{0}}{d r}\right)+R^{2} E_{0}^{2} B S_{c} U\left(S_{0}-S_{c}\right)=0 .
$$

The discontinuous term requires that Eq. (17) be solved in two parts; one for the electrically conducting core and the other for the annular

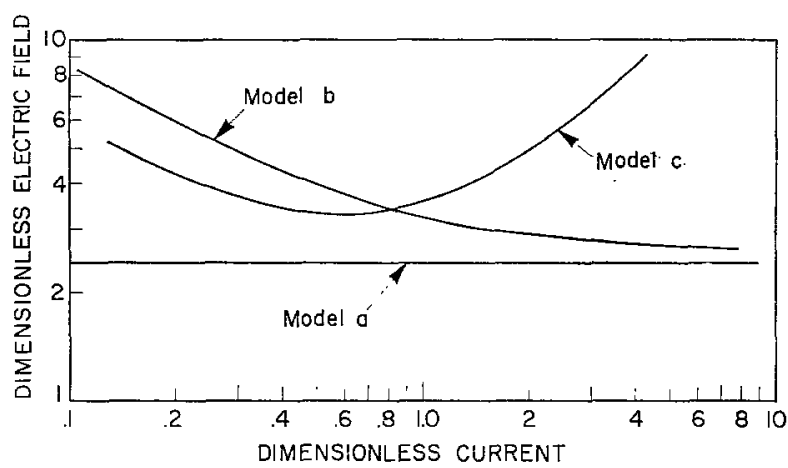

Fig. 3. Arc characteristics corresponding to approximate models

region. The two solutions must agree in slope and magnitude at the interface, leading to the result

$$
\begin{gathered}
S_{0}(r)=S_{c}\left[1+\frac{1}{4} R^{2} B E_{0}^{2}\left(r_{c}^{2}-r^{2}\right)\right], \quad 0 \leqq r \leqq r_{c}, \\
S_{0}(r)=S_{c} \frac{\ln r}{\ln r_{c}}, \quad r_{1} \leqq r \leqq 1 .
\end{gathered}
$$

Furthermore, from OHM's law we obtain

which leads to

$$
I_{0} / E_{0}=\pi R^{2} B S_{c} r_{c}^{2},
$$

$$
I_{0}^{2}=-4 \pi^{2} R^{2} B S_{c}^{2} r_{c}^{2} / \ln r_{c}^{2} .
$$

The $E-I$ characteristic for this model, as well as those for $(a)$ and $(b)$ is shown in Fig. 3. The current and electric field have been normalized with respect to the parameters that are appropriate to each model.

9 Z. Physik, Bd. 211 
The application of Eq. (11) to this model requires a little care because a derivative of the unit step function is involved. First we write

$$
\left(\frac{d \sigma}{d S}\right)_{S_{0}}=\left(\frac{d \sigma}{d r}\right)_{0} / \frac{d S_{0}}{d r}
$$

where $\left(d S_{0} / d r\right)$ is found from the DC solution above. Also,

$$
\left(\frac{d \sigma}{d r}\right)_{0}=-B S_{c} \delta\left(r-r_{c}\right),
$$

$\delta(r)$ being the Dirac delta function. There is no contribution to the integral in Eq. (11), except at $r=r_{c}$, and by integrating formally one obtains

$$
\theta_{g}=\frac{R^{2} r_{c}^{2}}{4 \lambda\left(S_{c}\right)}
$$

Besides being applicable to both high and low current arcs, Eq. (18), because of the sifting property of the delta function, includes the true variation of the thermal diffusivity. The selection of a value of $S_{c}$ for the model dictates the proper value of $\lambda$ to use in Eq. (18). Numerical values will be considered later.

The time constant for $S$ decay is obtained from Eq. (12) and the properties of the DC solution. There results, again for $r=0$,

$$
\left.\theta_{s}\right\}_{r=0}=\frac{R^{2} r_{c}^{2}}{4 \lambda(0)}\left[1+\frac{4 \pi S_{c}}{E_{0} I_{0}}\right]
$$

where $\lambda(0)$ is the thermal diffusivity at the center of the arc.

\section{The Effects of Induced Radial Flow}

In satisfaction of the continuity equation a radial velocity will always be induced in an arc whose properties vary with time. Radial convection has been ignored in the foregoing equations, as indeed it has in most other analyses of this type. WHITTAKER ${ }^{18}$ studied the effect of radial inflow on a free burning interrupted arc. Because only minute pressure gradients are required to drive the resulting flow, Whittaker ignored the momentum equation and obtained the radial velocity from the continuity equation. ChristmanN, Frie, and Hertz ${ }^{9}$ used the same method for a wall stabilized arc, but then one encounters difficulty with this approach because it is not possible to satisfy the zero velocity boundary condition at the tube wall. Furthermore, computing the radial flow from only the (1959).

${ }^{18}$ WhitTaker, D.: Proc. 4th Intl. Conf. on Ioniz. Phenom. in Gases, Uppsala 
continuity equation leads to the existence of a finite velocity immediately after arc interruption. It is shown here that this is not the case, that for the first moments after $t=0$ one has a constant density heat conduction problem. CLARKE ${ }^{19}$ found the same result for a related problem.

In order to assess the effects of convection it is necessary to consider the full set of conservation equations, Eqs. (1)-(4). Naturally, we make no attempt at a general solution to this system, rather we introduce the linearization concept of Sec. 3. Noting that there is neither a zeroth order velocity nor pressure gradient, it is a straightforward matter to obtain for the free decay problem with no radiation the following set of equations:

$$
\begin{gathered}
R \frac{\partial \rho^{\prime}}{\partial t}+\frac{1}{r} \frac{\partial}{\partial r}\left(\rho_{0} r v^{\prime}\right)=0 \\
R^{2} \rho_{0} \frac{\partial v^{\prime}}{\partial t}+R \frac{\partial p^{\prime}}{\partial r}-\frac{4}{3} \mu\left(S_{0}\right) \frac{\partial}{\partial r}\left(\frac{1}{r} \frac{\partial r v^{\prime}}{\partial r^{\prime}}\right) \\
-\frac{4}{3} \frac{d \mu\left(S_{0}\right)}{d r}\left(\frac{\partial v^{\prime}}{\partial r}-\frac{1}{2} \frac{v^{\prime}}{r}\right)=0 \\
\frac{R^{2}}{\lambda\left(S_{0}\right)} \frac{\partial S^{\prime}}{\partial t}+R^{2}\left[\rho_{0}\left(\frac{\partial h}{\partial p}\right)_{0}-1\right] \frac{\partial p^{\prime}}{\partial t}+\frac{R}{\lambda\left(S_{0}\right)} v^{\prime} \frac{d S_{0}}{d r} \\
+\sigma\left(S_{0}\right) R^{2} E_{0}^{2}-\frac{1}{r} \frac{\partial}{\partial r}\left(r \frac{\partial S^{\prime}}{\partial r}\right)=0
\end{gathered}
$$

$0 \leqq r \leqq 1, S^{\prime}(1, t)=0$. Again, the zero subscript solution is found from the stationary form of Eq. (7). The equation of state for the present problem becomes

$$
p(r, t)=p\left(S_{0}, \rho_{0}\right)+\left(\frac{\partial p}{\partial S}\right)_{\rho_{0}} S^{\prime}(r, t)+\left(\frac{\partial p}{\partial \rho}\right)_{S_{0}} \rho^{\prime}(r, t) .
$$

Clearly, what is required to close the set of Eqs. (19) - (21) is the relation

where

$$
p^{\prime}(r, t)=\alpha_{0}(r) S^{\prime}(r, t)+\beta_{0}(r) \rho^{\prime}(r, t)
$$

$$
\alpha_{0}(r)=\left(\frac{\partial p}{\partial S}\right)_{\rho_{0}} \text { and } \beta_{0}(r)=\left(\frac{\partial p}{\partial \rho}\right)_{S_{0}} .
$$

One can replace the spatial and temporal derivatives of $p^{\prime}$ with the above equation of state, leaving a set of three equations for the three dependent

${ }^{19}$ Clarke, J.F.: Rept. No. 124, Coll. Aeronautics, Cranfield (1960). 9* 
variables $v^{\prime}, S^{\prime}$, and $\rho^{\prime}$. These quantities are then represented as a power series in time, with radially dependent coefficients, i.e.

$$
\begin{aligned}
& v^{\prime}(r, t)=v_{1}(r) t+v_{2}(r) t^{2}+\cdots \\
& \rho^{\prime}(r, t)=\rho_{1}(r) t+\rho_{2}(r) t^{2}+\cdots .
\end{aligned}
$$

Consider first the expression for conservation of momentum, Eq. (20). Upon equating the coefficients of like powers of $t$, one finds that $v_{1}(r)=0$. Conservation of mass immediately yields $\rho_{1}(r)=\rho_{2}(r)=0$. Consequently, as noted earlier, the plasma decay process is initially one of constant density, the first density variation being $O\left(t^{3}\right)$. With no changes in density variation the continuity equation does not initially require the existence of a compensating inward flow. Then the initial variation of heat flux potential should proceed according to the classical conduction law. This is verified by what follows.

For the first term in the $S^{\prime}$ series one finds

$$
\left\{\frac{R^{2}}{\lambda\left(S_{0}\right)}+R^{2}\left[\rho_{0}\left(\frac{\partial h}{\partial p}\right)_{0}-1\right]\left(\frac{\partial p}{\partial S}\right)_{0}\right\} S_{1}(r)=-R^{2} \sigma\left(S_{0}\right) E_{0}^{2} .
$$

The terms in braces appear to be quite complex but an application of thermodynamic identities simplifies things considerably. The following development applies to any gas, with an arbitrary degree of dissociation and/or ionization. With the aid of a standard thermodynamics text (c.f. ZEMANSKY ${ }^{20}$ ) one can find

and

$$
\left(\frac{\partial p}{\partial S}\right)_{\rho}=\frac{1}{\bar{\kappa}}\left(\frac{\partial p}{\partial T}\right)_{\rho}=\frac{\rho a}{\bar{\kappa}} \sqrt{\frac{C_{p}-C_{v}}{T}}
$$

$$
\left[\rho\left(\frac{\partial h}{\partial p}\right)_{T}-1\right]=-\frac{1}{a} \sqrt{\gamma T\left(C_{p}-C_{v}\right)} .
$$

Here the only new symbols are $a$, the isentropic sound speed, $C_{v}$, the specific heat at constant volume, and $\gamma$, the ratio of specific heats. With the aid of these identities the expression in braces reduces to $R^{2} \rho C_{v} / \bar{\kappa}$, so that the coefficient of $S_{1}$ in the expression obtained previously is the inverse of a thermal diffusivity based upon $C_{v}$ rather than $C_{p}$. As a result the constant pressure theory developed in Sec. 3 can be corrected merely by replacing $\lambda\left(S_{0}\right)$ wherever it appears by $\lambda_{v}\left(S_{0}\right)$, where $\lambda_{v}=\bar{\kappa} / \rho C_{v}$. No other changes are necessary. This finding underscores the fact that for the first moments after arc interruption the pressure variations in the column cannot be ignored; they account for the existence of constant

${ }^{20}$ Zemansky, M.W.: Heat and thermodynamics, 4th ed. New York: McGrawHill Book Co. 1957. 
volume heat conduction. Indications are that this situation prevails for some time after interruption, at least until the density starts changing. This can be shown by a comparison of time constants for pressure and heat flux potential at $t=0$. These can be determined by applying the method of Sec. 3 to the equations for $S^{\prime}$ and $p^{\prime}$. The resulting expressions are

$$
\theta_{s}=S_{0}(r) / S_{1}(r), \quad \theta_{p}=p_{0} / \alpha_{0}(r) S_{1}(r)
$$

The ratio of time constants $\theta_{p} / \theta_{s}$ is simply $p_{0} / \alpha_{0} S_{0}$. This has been evaluated at several values of $r$ for both argon and nitrogen arcs and was found to be of order unity. Thus one cannot, initially at least, assume that the pressure is constant and compute the induced velocity from the continuity equation alone. After a while the plasma will decay at essentially constant pressure, but by that time there is no longer an induced velocity. A more detailed treatment of this problem, including the effects of convection at later times after interruption, is discussed by PhILLIPs ${ }^{21}$.

\section{Application to Experimental Results}

\subsection{General}

Very little data exists with which the present theories can be compared. The measurements of YOON and SPINDLE ${ }^{11}$, for instance, were made on a low current arc burning in a $3 / 4$ in. diameter tube, conditions which classify the arc more as free burning than wall-stabilized. More pertinent is the data of Christmann, Frie, and Hertz ${ }^{9}$ and Kimblin and Edels ${ }^{10}$. The former investigators have measured the variation in conductance following the interruption of $5 \mathrm{~mm}$ diameter argon and nitrogen arcs, at various current levels. The latter work is concerned with similar measurements on a $5 \mathrm{~mm}$ air arc at currents up to 36 amperes. Both investigations show that for the diatomic gases the conductance appears to decay with two time constants; the first is about $10 \mu \mathrm{sec}$ while the terminal value is of the order of $100 \mu \mathrm{sec}$. In argon, Christmann, Frie, and Hertz ${ }^{9}$ found that the conductance decay proceeded with a single time constant, about $120 \mu \mathrm{sec}$. Since the present theory applies to only the initial stages of free recovery, it cannot be expected to predict the behavior of the entire decay process, including the appearance of two or more time constants. One should, however, be able to predict the value of initial time constants and their variation with initial current. In what remains, then, we shall obtain some numerical values for the expressions developed in Sec. 3 and 4 and compare them with appropriate experimental data.

${ }^{21}$ Phillirs, R.L.: Proc, 8th Intl. Conf. on Phenom. in Ioniz. Gases, Vienna (1967). 


\subsection{Free Recovery of the Interrupted DC Arc}

6.2.1. Influence of Type of Gas. Recall from Sec. 3 that in its most general form the evaluation of $\theta_{\mathrm{g}}$ from Eq. (11) requires a numerical integration over the arc cross section of the product of three complicated functions. In particular, the thermal diffusivity is involved, requiring a knowledge of an imperfectly known transport property, $\bar{\kappa}$, the thermal conductivity. It is therefore interesting that one can effect a transformation that obviates the need to know $\bar{\kappa}$. To show this, consider the new variable $F$, defined by the relation

$$
d F=\rho C_{v} d T .
$$

From the definition of the heat flux potential, $\bar{\kappa}=d S / d T$, so the thermal diffusivity can be expressed as

$$
\lambda_{v}=\frac{\bar{\kappa}}{\rho C_{v}}=\frac{d S}{d T} \cdot \frac{d T}{d F} .
$$

Thus, the integrand in Eq. (5) can be transformed into a function of $F$, allowing $\theta_{g}$ to be written as

$$
\theta_{\mathrm{g}}^{-1}=-\frac{\pi R^{2} E_{0}^{3}}{I_{0}} \int_{0}^{1} r\left(\frac{d \sigma^{2}}{d F}\right)_{0} d r
$$

The identity $E_{0} G_{0}=I_{0}$ has been used to obtain Eq. (22) and, as usual, the subscript ( $)_{0}$ refers to properties of the initial state of the plasma. Unlike $\bar{\kappa}$, the thermodynamic properties $\rho$ and $C_{v}$ are known with reasonable certainty ${ }^{22}$, as is $\sigma$, the electrical conductivity ${ }^{23,24}$. For a pressure of one atmosphere the function $\sigma^{2}(F)$ is plotted in Fig. 4 for both nitrogen and argon. The striking geometrical similarity of the curves has important implications because the integrand in Eq. (22) involves only the slope of the $\sigma^{2}(F)$ function. Since this slope is much smaller at low temperatures than at high, one can approximate the integral by a constant, weighted with respect to radius. The similarity of the curves implies that this constant is nearly the same for both gases. Moreover, for an initial current of 100 amperes, the maximum temperature in a $5 \mathrm{~mm}$ diameter arc is about $14,000^{\circ} \mathrm{K}$ for argon as well as nitrogen. Thus, the areal weighting factors for the two integrals are very nearly equal, indicating that the essential difference between argon and nitrogen time constants is due to $E_{0}$, the initial field strength. Indeed, when the ratio of the field strengths for 100 ampere argon and

22 Drellishak, K.S., D.P. Aeschliman, and A.B. CAmbel: Rept. No. AEDCTDR-64-12, Arnold Eng. Dev. Ctr. (1964).

${ }^{23}$ Yos, J.: AVCO Tech. Memo RAD-TM-63-7 (1963).

24 Devoto, R.S.: Phys. Fluids 10, 354 (1967). 
nitrogen arcs (as computed by PATT ${ }^{25}$ ) is raised to the third power, one obtains the ratio of initial time constants $(120 \mu \mathrm{sec} / 18 \mu \mathrm{sec})$ found by ChristManN, Frie, and Hertz ${ }^{9}$. It is conjectured therefore, that a rapid method for predicting the relative rates of dielectric recovery for some

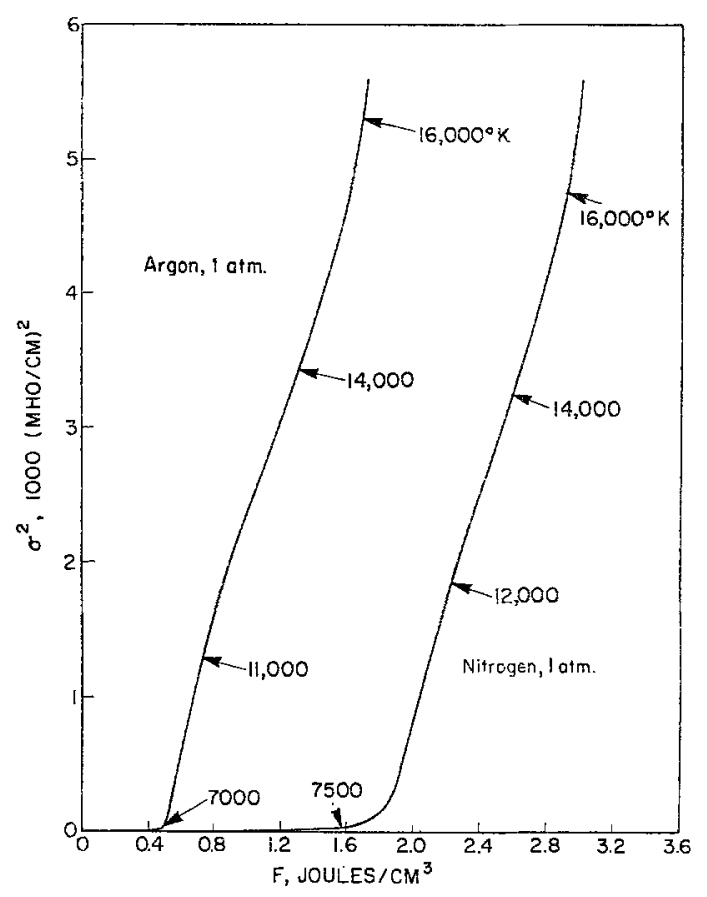

Fig. 4. Electrical conductivity function vs. Energy density integral

gases might be to form the cube of the ratios of $E_{0}$ for selected current levels. An $E_{0}-I_{0} \mathrm{DC}$ characteristic is relatively easy to obtain, allowing one to assess the interrupting value of any gas by quite unsophisticated methods.

6.2.2. Influence of Current Level (Nitrogen). FrIND ${ }^{6}$ predicted, semiempirically, the behavior of the conductance time constant with initial arc current. Depending upon the gas in question he finds that $\theta_{g}$ first increases with current, then falls to some minimum value, and subsequently rises indefinitely with increasing current. The present theory (Eq. (22)) can be used without resorting to empiricism and it is interesting to see that the results compare well, at least qualitatively, with those of FRIND.

${ }^{25}$ PATT, H.-J.: Zur Plasmadynamik in wandstabilisierten Lichtbogen. Thesis, Aachen (1964). 
In order to avoid using the imperfectly known nitrogen thermal conductivity, initial temperature distributions and electric field strengths that were measured ${ }^{26}$ rather than calculated have been used. Since the integral in Eq. (22) involves a single valued function of temperature, it can be evaluated numerically for each value of initial current. This has

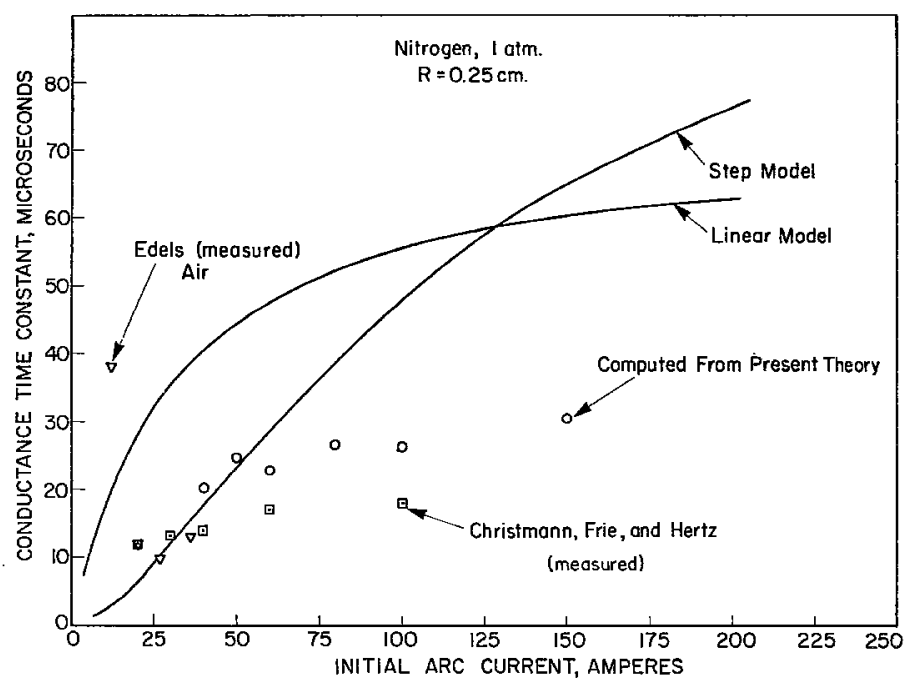

Fig. 5. Comparison of present theory with measurements

been done for nitrogen at one atmosphere, using the sources of material properties mentioned earlier. The results are shown in Fig. 5. In addition, the time constant curves resulting from the simplified models (b) and (c) from Sec. 4 are shown. The constants $B, S_{c}$, and $\lambda_{0}$ were chosen to provide a best fit to measured characteristics ${ }^{27}$. Finally, the measured time constants of Christmann, Frie, and Hertz ${ }^{9}$ and Kimblin and EDELS ${ }^{10}$ are indicated for comparison. The latter measurements, recall, are for air.

It is not surprising that the approximate models do not agree with the measurements or the accurate theory; too many simplifications are necessary in order to obtain closed form results.

Christmann, Frie, and Hertz ${ }^{9}$ have measured $\theta_{g}$ for initial currents of $20,30,40,60$, and 100 amps. It is seen that these points fall consistently below the present theory. Since the theory is quite complete this discrepancy could indicate a deficiency in the values of electrical conductivity $^{23}$ that were used in the computation. Of course, the various

${ }^{26}$ Natl. Aero. and Space Admin. Rept. CR-575 (1966).

${ }^{27}$ MAECKER, H.: Z. Physik 158, 392 (1960). 
measurements of temperature profile, $E_{0}$, and $\theta_{g}$ could be in error, but the corresponding investigators claim good accuracy for their results.

It is possible that LTE does not prevail in the experiments, especially near $t=0$, but this seems unlikely when one calculates typical equilibration times for the various processes occurring in the plasma.

Finally, the possibility of axial velocity effects should be considered. The theory, recall, was developed for an infinitely long cylinder while the actual experimental arc will be subject to end effects. Just how soon after modulation axial pressure relief will occur depends upon the length to diameter ratio of the arc column ( $L / D \cong 44$ for the cascade of Ref. 9). For times immediately after modulation, note that end effects would not alter the present theory because the induced axial flow requires some time to develop, just as with the radial flow discussed earlier. Actual arc modulation, however, cannot be effected in times much less than one microsecond. The question of the importance of axial flow, then, reduces to a comparison of experimental times versus theoretical times and further work is required before this point can be clarified.

\section{Concluding Remarks}

A theory has been developed that allows one to compute the time constants for various arc parameters shortly after the interruption or step modulation of the sustaining current. Numerical results were presented for $\theta_{g}$ using both simplified transport properties and the best available exact values for nitrogen.

Extention of the present theory is proceeding along the following lines:

1. Inclusion of radiative transfer effects, i.e., a nontransparent plasma.

2. Consideration of longer times after current modulation, including the effects of radial convection.

3. Consideration of more complicated boundary conditions (free burning and blown ares) and current modulation functions.

4. Examination of terminal time constants in order to predict the rates at which final states are approached.

Finally it should be mentioned that experiments with a specially designed cascade arc chamber are now under way which will provide the additional data necessary to verify the present and future analyses.

Dr. RICHARd L. PHILLIPS

University of Michigan

Dept. of Aerospace Engineering

Ann Arbor, Michigan/USA 